\title{
A SHAPE-TEMPLATE BASED TWO-STAGE CORPUS CALLOSUM SEGMENTATION TECHNIQUE FOR SAGITTAL PLANE T1-WEIGHTED BRAIN MAGNETIC RESONANCE IMAGES
}

\author{
Jayanth Krishna Mogali ${ }^{1}$, Naren Nallapareddy ${ }^{1}$, Chandra Sekhar Seelamantula ${ }^{1}$, Michael Unser ${ }^{2}$ \\ ${ }^{1}$ Department of Electrical Engineering, Indian Institute of Science, Bangalore \\ ${ }^{2}$ Biomedical Imaging Group, École polytechnique fédérale de Lausanne, Lausanne, Switzerland \\ E-mails: \{jayanth.mogili, naren.mit12\}@gmail.com, chandra.sekhar@ieee.org, michael.unser@epfl.ch
}

\begin{abstract}
We propose a semi-automatic technique to segment corpus callosum (CC) using a two-stage snake formulation: A restricted affine transform (RAT) constrained snake followed by an unconstrained snake in an iterative fashion. A statistical model is developed to capture the shape variations of CC from a training set, which restrict the unconstrained snake to lie in the shape-space of CC. The geometry of the constrained snake is optimized using a local contrast-based energy over RAT space (which allows for five degrees of freedom). On the other hand, the unconstrained snake is optimized using a unified energy (region, gradient, and curvature energy) formulation. Joint optimization resulted in increased robustness to initialization as well as fast and accurate segmentation. The technique was validated on 243 images taken from the OASIS database and performance was quantified using Jaccard's distance, sensitivity, and specificity as the metrics.
\end{abstract}

Index Terms - Corpus callosum segmentation, active contour model, shape-specific snake, contrast-based energy.

\section{INTRODUCTION}

Corpus callosum (CC) is a broad, transverse neural pathway in the mammalian brain, which serves as a connection between the two cerebral hemispheres [1]. There is a vast body of literature in neurobiology that connects its morphological features such as shape and size to sexual dimorphism [2], handedness [2], and pathologies such as dyslexia [3], schizophrenia [4], autism [5], epilepsy [6], and multiple sclerosis [7]. Therefore, finding the anatomical features of CC forms an important stage in diagnosis. For imaging the brain, Magnetic Resonance Imaging (MRI) is preferred as it provides better contrast between different soft tissues than traditional radiography or computed tomography [8].

This work was sponsored by the Department of Science and Technology (DST) - Intensive Research in High Priority Areas (IRHPA) of the Government of India (Project code: DSTO-943).
Snakes are a popular image segmentation tool [9], which grow from a specific initialization and converge on to boundary of interest under the influence of certain image and internal forces. Image forces (typically region and gradient energy) direct the contour to the boundary of interest, and internal forces ensure a certain degree of smoothness or regularity to the curve. Since their introduction by Kass et al. [9], many modifications have been proposed in snake design, the novelty of which generally lies in specification of curve representation and suitable energies. Curve representations such as B-splines [9,10], Fourier descriptors [11], wavelets [12], etc. have been developed to solve specific segmentation problems. B-splines, in particular, are inherently smooth, have compact support, and provide local control, which make them a popular choice for medical image segmentation. Previous work on segmentation incorporating shape information in the Bspline framework include $[13,14]$. In our problem, $C C$ has a distinguishable shape that makes it a suitable candidate for incorporating shape information into the segmentation algorithm $[15,16]$. On the energy front, many techniques rely on region-and gradient-based energies or functions derived thereof [17]. Recently, Thévenaz and Unser [18,19] proposed a new contrast-based energy for optimizing concentric circles and ellipses. The contrast is defined between the pixel intensities inside the inner contour and those lying in the annular region between the inner and outer contours. In a T1-weighed sagittal plane MR image of the brain, CC can be seen as a light gray non-convex structure locally brighter than its surroundings. The contrast between $\mathrm{CC}$ and surrounding regions has motivated us to use contrast energy for our snake formulation.

In this paper, we propose a hybrid strategy that combines a shape-specific parametric snake optimized over affine space followed by an unconstrained parametric snake optimization. The unconstrained snake deformation is monitored every few iterations and is restricted to lie in the shape-space of the CC. The segmentation comprises two major stages:

1. Affine-space optimization: In this stage, a snake consisting of a nested template is parameterized using cubic B-splines (called a B-snake) [13]. The snake is 
manually initialized and is allowed to deform in the RAT space of the template. The snake energy used is the contrast-based energy introduced in $[18,19]$. This stage results in a coarse but fast segmentation. The details are given in Section 2.1 .

2. Shape-space optimization: The converged snake from the first stage is used as an initialization for a unifiedenergy (gradient, curvature, and region energies) snake [10], which refines the segmentation. This snake is periodically projected on to the shape-space of CC every few iterations (100 in the experiments reported) and restricted to lie within it.

If the snake in stage 2 converges within a predefined number of iterations, the process is deemed complete. Otherwise, a new template is derived from stage 2 and used in stage 1 as initialization (automatic), the two stages are repeated until the process is complete.

The rest of the paper is organized as follows. In Section 2, we present the details of our proposed algorithm with associated background materials, which we follow up with segmentation results in Section 3. In Section 4, we present conclusion and scope for extending the proposed technique.

\section{PROPOSED FORMULATION}

\subsection{Affine-space snake formulation}

In the first stage, we use a deformable template-based segmentation approach, utilizing a contrast-based energy for optimization. The deformable template consists of a pair of nested contours and allowed to move in the template's RAT space. Thévenaz and Unser [18,19] employed a shapespecific parametrization and regularization to maintain shape specificity. In [20], we provided a unified approach for segmentation of circular and elliptical shaped objects. In [21], we adapted the approach to segment rectangular shaped objects and showed applications to western blot image analysis. In this paper, we extend the approach to deal with the more complex shape of corpus callosum.

\subsubsection{Template parametrization}

The inner and outer contours derived from the shape template, $\mathbf{r}_{0}(t)=\left(x_{0}(t), y_{0}(t)\right)^{T}$ and $\mathbf{r}_{1}(t)=\left(x_{1}(t), y_{1}(t)\right)^{T}$, where $t$ is an independent variable, are reparametrized as a linear combination of cubic B-splines. The contours are closed and here it follows that $x_{i}(t)$ and $y_{i}(t)$ are periodic $[10,22]$. Accordingly, we have:

$$
\mathbf{r}_{i}(t)=\left(\begin{array}{l}
x_{i}(t) \\
y_{i}(t)
\end{array}\right)=\sum_{k=0}^{M-1} \mathbf{c}_{i, k} \beta_{M}(t-k),
$$

where $\mathbf{c}_{i, k}=\left(\begin{array}{l}c_{x_{i}, k} \\ c_{y_{i}, k}\end{array}\right)$ denote the spline coefficients, $\beta_{M}$ is the $M$-periodic version of the cubic $\mathrm{B}$-spline basis $\beta$.

\subsubsection{From template to snake}

Snakes are derived from the parametrized template using the following transformation:

$$
\left(\begin{array}{c}
X_{i}(t) \\
Y_{i}(t)
\end{array}\right)=\underbrace{\left(\begin{array}{ccc}
A \cos \theta & B \sin \theta & x_{c} \\
-A \sin \theta & B \cos \theta & y_{c}
\end{array}\right)}_{\mathbb{M}}\left(\begin{array}{c}
x_{i}(t) \\
y_{i}(t) \\
1
\end{array}\right),
$$

where $i=0,1$, and $\left(X_{0}(t), Y_{0}(t)\right)$ and $\left(X_{1}(t), Y_{1}(t)\right)$ represent the coordinates of the inner and outer contours of the snake, respectively. $A$ and $B$ represent the scale factors in directions perpendicular and axial to the $\mathrm{CC}$, respectively, $\theta$ represents the angle of rotation (clockwise), and $\left(x_{c}, y_{c}\right)$ are the translation parameters. The spline coefficients of the transformed snake are obtained by applying the transformation $\mathbb{M}$ to the spline coefficients of the template [23]. Having defined the contour parametrization and its transformation over RAT space, we move on to the training phase of the algorithm.

\subsubsection{Training}

We annotated ${ }^{1}$ a training set of images manually by placing knots along the $\mathrm{CC}$ boundary. The annotated contours are aligned by minimizing the $L_{2}$ norm between them. Consider two curves $\Omega_{1}$ and $\Omega_{2}$, which are aligned by minimizing the distance $D$ with respect to $\left\{A, B, \theta, x_{c}, y_{c}\right\}$ as:

$$
D=\left\|\Omega_{1}-\mathbb{M}\left(A, B, \theta, x_{c}, y_{c}\right) \Omega_{2}\right\|_{L_{2}} .
$$

Fortunately, due to the Riesz basis property of B-splines [23], minimization of the $L_{2}$ norm in (3) is equivalent to minimization of a discrete sum with the following inequality:

$$
D \leq \gamma\left\|C_{\Omega_{1}}-\mathbb{M}\left(A, B, \theta, x_{c}, y_{c}\right) C_{\Omega_{2}}\right\|_{\ell_{2}}
$$

where $\gamma$ is the upper Riesz bound and $C_{\Omega_{1}}, C_{\Omega_{2}}$ represent the spline coefficients of $\Omega_{1}$ and $\Omega_{2}$, respectively. The rest of the procedure for aligning the curves in the training set is similar to Procrustes analysis [24]. Once the alignment is completed, PCA is applied on the spline coefficients of the aligned curves to obtain the mean and eigenvectors of the covariance matrix. This constitutes our statistical model for the CC.

\footnotetext{
${ }^{1}$ Twenty six knots were placed on contour for annotation purposes. Closely spaced knots were placed along the posterior and middle Splenium sections to capture the high curvature. In the remaining sections, approximately equidistant knots were placed.
} 


\subsubsection{Affine-space optimization}

Motivated by the definition of contrast energy introduced in $[18,19]$, we propose a modified energy:

$$
\begin{aligned}
\mathscr{E} & =\frac{1}{A B}\left\{a_{0} \iint_{\Re_{1} \backslash \Re_{0}} f \mathrm{~d} x \mathrm{~d} y-\left(a_{1}-a_{0}\right) \iint_{\Re_{0}} f \mathrm{~d} x \mathrm{~d} y\right\} \\
& =\frac{1}{A B}\left\{a_{0} \iint_{\Re_{1}} f \mathrm{~d} x \mathrm{~d} y-a_{1} \iint_{\Re_{0}} f \mathrm{~d} x \mathrm{~d} y\right\}
\end{aligned}
$$

where $a_{0}$ and $a_{1}$ are the areas of inner and outer templates, respectively. $\Re_{0}$ and $\Re_{1}$ are the regions enclosed by the inner and outer contours ${ }^{1}$ of the snake, respectively; $f$ is the image on which the contour lies. Minimizing $\mathscr{E}$ over the RAT space enables the snake to lock on to objects that are brighter than their surroundings. The primary role of the outer contour is that it guides computation of contrast in a local fashion.

We proceed to optimizing the energy with respect to RAT parameters $\left\{A, B, \theta, x_{c}, y_{c}\right\}$. Given an initialization for the RAT parameters, we employ gradient descent optimization technique to obtain the optimal values. Partial derivatives are required to the solve the problem; hence, we follow a procedure similar to that of $[20,21]$ where the surface integrals are transformed to contour integrals using Green's theorem. Owing to space constraints we only provide the final expressions in (6) and (7).

\subsection{Shape-space optimization}

The output of the affine space optimization is used as the initialization in this stage. The snake is allowed to deform in an unconstrained manner, which is necessary because the snake in the first stage may not converge to provide an optimal fit to the boundaries. This behavior can be attributed to initialization or shape mismatch between the template used and actual CC contour. In our algorithm, we chose unified energies (based on region, gradient, and curvature) [10] for snake optimization. In addition, we periodically constrain the deformation to lie within the shape space model of $\mathrm{CC}$ by using a projection technique explained in the next paragraph. The methodology is similar to that of [13] except that we use restricted affine space and do not restrict the snake after every iteration.

The snake is projected on to the space spanned by the significant eigenvectors obtained from PCA. A procedure similar to active shape model [24] in affine space is used wherein the components exceeding the ellipsoidal manifold are truncated to the nearest point on the surface. The principal axes (eigenvectors) of the ellipsoidal manifold are of length $6 \sqrt{\lambda}$, where $\lambda$ is the eigenvalue corresponding to the eigenvectors spanning the space.

\footnotetext{
${ }^{1}$ The outer contour is obtained by segmenting the region formed by dilating the flood-fill output of inner contour. We chose a circular kernel of radius 10 pixels for dilation.
}

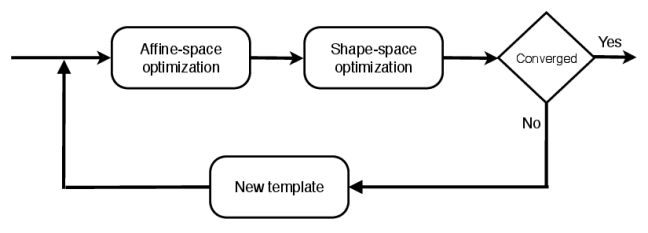

Fig. 1. Flowchart of the proposed approach.

\section{RESULTS AND DISCUSSIONS}

Test images were taken from OASIS database [25], which contains 315 images out of which 72 images were used for training and 243 images for validation. For all images, the initialization $^{2}$ provided was similar to that shown in Fig. 3(a). The average time for convergence was found to be 219.687 ms with a standard deviation of $169.69 \mathrm{~ms}$. In about $10 \%$ of the cases, mismatch in shape between the actual CC contour and the mean template resulted in multiple passes of stages 1 and 2. However, in a majority of the cases, it converged to the optimal fit in one or two passes. The experiment was performed using ImageJ software on an Intel Core ${ }^{2}$ Quad CPU running at $2.8 \mathrm{GHz}$.

Another experiment was conducted to show the effect of initialization on convergence (cf. Fig. 3). We note that in Fig. 3(a)-(e), the initialization resulted in segmentation output given in Fig. 3(f). We used Jaccard's distance (JD), sensitivity, and specificity to measure the performance of the algorithm on 243 images using the atlas provided in the database. The mean values of JD, sensitivity, and specificity obtained are $0.914,0.921$, and 0.996 , respectively.

The fast convergence of the algorithm can be attributed to the template-based optimization stage. Only five parameters are optimized for irrespective of the number of knots parameterizing the contour. This resulted in reduced computational requirements. Moreover, the two stages work in a synergistic fashion, the advantage being increased robustness to initialization and better segmentation performance. Unconstrained snakes might be misled by neighboring structures and are prone to looping. Enforcing shape constraints such as those employed in [13], requires costly matrix inversion operations making it computationally expensive. In contrast, we operate in the affine space and shape space alternatively, which leads to faster convergence.

\section{CONCLUSION}

We proposed a two-stage corpus callosum segmentation technique where in we incorporate explicit shape constraints using a restricted affine transformation with five degrees of freedom. The five parameters are optimized for using local con-

\footnotetext{
${ }^{2}$ The template used for manual initialization is the mean template obtained from the training set.
} 


$$
\begin{gathered}
\left(\begin{array}{c}
\partial \mathscr{E}_{i} / \partial A \\
\partial \mathscr{E}_{i} / \partial B \\
\partial \mathscr{E}_{i} / \partial \theta
\end{array}\right)=\frac{\alpha_{i}}{A B} \sum_{k, l=0}^{M-1} R_{f}(k, l)\left(\begin{array}{c}
B c_{x_{i}, k} c_{y_{i}, l} \\
-A c_{x_{i}, l} c_{y_{i}, k} \\
A^{2} c_{x_{i}, k} c_{x_{i}, l}+B^{2} c_{y_{i}, k} c_{y_{i}, l}
\end{array}\right)-\left(\begin{array}{c}
\frac{1}{A_{1}^{2} B} \\
\frac{1}{A B^{2}} \\
0
\end{array}\right) \mathscr{E} \\
\left(\begin{array}{c}
\partial \mathscr{E}_{i} / \partial x_{c} \\
\partial \mathscr{E}_{i} / \partial y_{c}
\end{array}\right)=\frac{\alpha_{i}}{A B} \sum_{k=0}^{M-1}\left(\begin{array}{c}
-A \sin \theta c_{x_{i}, k}+B \cos \theta c_{y_{i}, k} \\
-A \cos \theta c_{x_{i}, k}-B \sin \theta c_{y_{i}, k}
\end{array}\right) \times S_{f}(k),
\end{gathered}
$$

where $R_{f}(k, l)=\int_{0}^{M} f\left(X_{1}(t), Y_{1}(t)\right) b_{k, l}(t) \mathrm{d} t$, and $b_{k, l}(t)=\beta_{P}(t-k) \beta_{P}^{\prime}(t-l), M$ denotes the number of knots. $S_{f}(k)=\int_{0}^{M} f\left(X_{1}(t), Y_{1}(t)\right) \beta_{P}^{\prime}(t-k) \mathrm{d} t . \alpha_{i}= \begin{cases}a_{1} & \text { if } i=0, \\ a_{0} & \text { if } i=1 .\end{cases}$
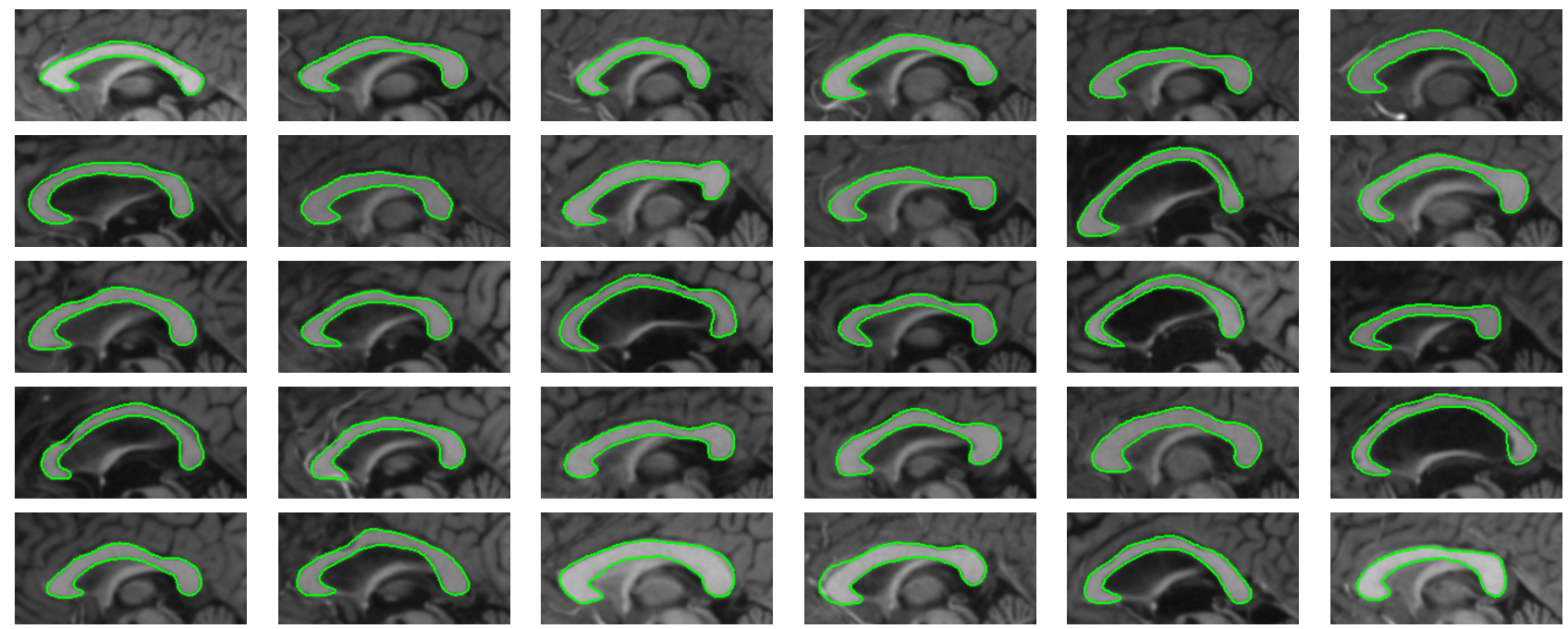

Fig. 2. Segmentation results using the proposed algorithm for some representative images taken from the database. We have chosen images that have a large curvature or having a diffused edge between CC contour and fornix or both.

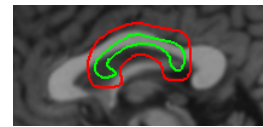

(a)

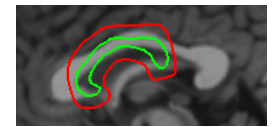

(b)

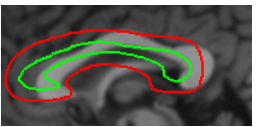

(c)

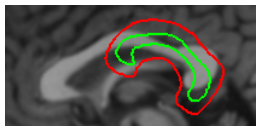

(d)

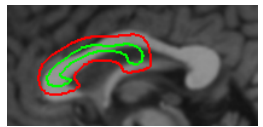

(e)

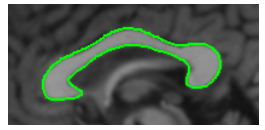

(f)

Fig. 3. Effect of initialization: Images (a)-(e) show various initializations, all of which converge to the result shown in (f).

trast as the energy function. This step gives rise to a fast, but coarse segmentation, which is further refined by shapespace optimization, wherein a deformable parametric active contour is evolved with restrictions to belong to a shape space derived out of training set of images. The segmentation performance turned out to be quite reliable over a large number of images taken from the OASIS database indicating that such a two-pronged strategy is robust to various shapes forms and deviations from the average shape structure. The segmentation performance is also quite robust to initialization and does not get deviated by the fornix, which usually poses problems in segmentation of corpus callosum. The proposed methodology is image-specific, that is, the training images guide the shape-space optimization. The advantage of this approach is that the shape-space optimization part can be tuned to a desired class of images, whereas the affine-space optimization remains relatively unchanged. 


\section{REFERENCES}

[1] J. S. Bloom and G. W. Hynd, "The role of the corpus callosum in interhemispheric transfer of information: Excitation or inhibition?," J. Neuropsychology Rev., vol. 15, pp. 59-71, January 2005.

[2] M. Habib, D. Gayraud, A. Olivia, J. Regis, G. Salamon, and R. Khalil, "Effects of handedness and sex on the morphology of the corpus callosum: A study with brain magnetic resonance imaging," J. Brain Cognition, vol. 16, no. 1, pp. 41-61, 1991.

[3] J. M. Rumsey, M. Casanova, G. B. Mannheim, N. Patronas, N. De Vaughn, S. D. Hamburger, and T. Aquini, "Corpus callosum morphology, as measured with MRI, in dyslexic men," J. Biological Psychiatry, vol. 39, no. 9, pp. 769-775, May 1996.

[4] J. E. Downhill, Jr. M. S. Buchsbaum, T. Wei, J. SpiegelCohen, E. A. Hazlett, M. M. Haznedar, J. Silverman, L. J. Siever, "Shape and size of corpus callosum in schizophrenia and schizotypal personality disorder," $J$. Schizophr. Res., vol. 42, no. 3, pp. 193-208, 2000.

[5] B. Egaas, E. Courchesne, and O. Saitoh, "Reduced size of corpus callosum in autism," Archives Neurobiology, vol. 52, no. 8, pp. 794-801, 1995.

[6] B. Weber, E. Luders, J. Faber, S. Richter, C. M. Quesada, H. Urbach, P. M. Thompson A. W. Toga, C. E. Elger, and C. Helmstaedter, "Distinct regional atrophy in the corpus callosum of patients with temporal lobe epilepsy," J. Brain, vol. 130, pp. 3149-3154, Dec 2007.

[7] R. O. Barnard and M. Triggs, "Corpus callosum in multiple sclerosis," J. Neurology, Neurosurgery, and Psychiatry, vol. 37, no. 11, pp. 1259-1264, 1974.

[8] R. R. Edelman and S. Warach, "Magnetic resonance imaging," New England J. Medicine, vol. 328, no. 10, pp. 708-716, 1993.

[9] M. Kass, A. Witkin, and D. Terzopoulos, "Snakes: Active contour models," Int. J. Comp. Vision, vol. 1, no. 4, pp. 321-331, 1988.

[10] M. Jacob, T. Blu, and M. Unser, "Efficient energies and algorithms for parametric snakes," IEEE Trans. Image Process., vol. 13, no. 9, pp. 1231-1244, 2004.

[11] E. Person and $\mathrm{S}$. Fu, "Shape discrimination using Fourier descriptors," IEEE Trans. Syst. Man Cybern., vol. 7, no. 3, pp. 170-179, 1977.

[12] Q. M. Tueng and W. W. Boles, "Wavelet-based affine invariant representation: A tool for recognizing planar objects in 3D space," IEEE Trans. Patt. Anal. Mach. Intell., vol. 19, pp. 846-857, 1997.
[13] Y. Wang and E. K. Teoh, "Dynamic B-snake model for complex objects segmentation," Image and Vision Computing, vol. 23, pp. 1029-1040, 2005.

[14] C. Tejos, L. D. Hall, and A. Cardenas-Blanco, "Segmentation of articular cartilage using active contours and prior knowledge," Proc. Ann. Int. Conf. IEEE Eng. Medicine and Biology Soc., vol. 1, 2004.

[15] C. Lee, S. Huh, T. A. Ketter, and M. Unser, "Automated segmentation of the corpus callosum in midsagittal brain magnetic resonance images," J. Optical Eng., vol. 39, pp. 924-935, 2000.

[16] C. Vachet, B. Yvernault, K. Bhatt, R. G. Smith, G. Gerig, H. C. Hazlett, and M. Styner, "Automatic corpus callosum segmentation using a deformable active Fourier contour model," Proc. SPIE Med. Imag.: Biomed. Appl. Molecular Structural Functional Imag., vol. 8317, pp. 831707, 2012.

[17] C. Xu and J. L. Prince, "Snakes, shapes, and gradient vector flow," IEEE Trans. Image Process., vol. 7, no. 3, pp. 359-369, 1998.

[18] P. Thévenaz and M. Unser, "Snakuscules," IEEE Trans. Image Process., vol. 17, no. 4, pp. 585-593, 2008.

[19] P. Thévenaz, R. Delgado-Gonzalo, and M. Unser, "The ovuscule," IEEE Trans. Pattern Anal. Mach. Intell., vol. 33, no. 2, pp. 382-393, 2010.

[20] A. K. Pediredla and C. S. Seelamantula, "A unified approach for optimization of snakuscules and ovuscules," Proc. IEEE Int. Conf. on Acoustics, Speech, and Signal Process., pp. 681-684, 2012.

[21] A. K. Pediredla and C. S. Seelamantula, "Activecontour-based automated image quantitation techniques for western blot analysis," Proc. IEEE rth Intl. Symp. Image and Sig. Proc. Anal., pp. 331-336,2011.

[22] P. Brigger, J. Hoeg, and M. Unser, "B-spline snakes: A flexible tool for parametric contour detection," IEEE Trans. Image Process., vol. 9, no. 9, pp. 1484-1496, 2000.

[23] M. Unser, "Splines: A perfect fit for signal and image processing," IEEE Signal Process. Mag., vol. 16, no. 6, pp. 22-38, 1999.

[24] T. Cootes, "An introduction to active shape models," Image Process. Anal., pp. 223-248, 2000.

[25] D. S. Marcus, T. H. Wang, J. Parker, J. G. Csernansky, J. C. Morris, and R. L. Buckner, "Open access series of imaging studies (OASIS): Cross-sectional MRI data in young, middle aged, non-demented, and demented older adults," J. Cognitive Neuroscience, vol. 19, no. 9, pp. 1498-1507, 2007. 